( О.Д. Рымар, С.В. Мустафина, В.И. Алфёрова*, Д.В. Денисова

Научно-исследовательский институт терапии и профилактической медицины - филиал Института цитологии и генетики Сибирского отделения Российской академии наук, Новосибирск, Россия

АКТУАЛЬНОСТЬ. По данным Глобальной сети по йоду, Российская Федерация является регионом умеренного дефицита йода. В настоящее время в России отсутствует системный контроль за состоянием йододефицита в регионах. Существующее законодательство устанавливает добровольный характер йодной профилактики. В связи с этим актуальным является вопрос изучения обеспеченности йодом населения Сибирского региона на примере г. Новосибирска.

ЦЕЛЬ. Оценить обеспеченность йодом и эффективность профилактики йодного дефицита среди подростков 13-18 лет и лиц молодого возраста 19-25 лет г. Новосибирска.

МАТЕРИАЛЫ И МЕТОДЫ. В исследование были включены репрезентативные выборки школьников 13-18 лет (612 человек обоего пола, из которых 62 человека заполнили опросник об использовании йодированной соли, у 60 из них были взяты образцы мочи для определения концентрации йода в моче (КЙМ)) и молодых людей 19-25 лет (101 человек обоего пола, все они заполнили предлагаемый опросник, у 27 человек была определена КЙМ). Проводились анкетирование, определение КЙМ, сопоставление полученных данных с результатами клинико-популяционных исследований, проводимых в г. Новосибирске в 1994-1995, 2004-2005, 2009-2010 гг.

РЕЗУЛЬТАТЫ. В 2005 г. потребляли йодированную соль 32,7\% взрослого населения. В 2010 г. $47 \%$ опрошенных подростков потребляли йодированную соль. По данным анкетирования, проведенного в 2019 г., среди взрослых 19-25 лет 19,8\% опрошенных знают о том, что потребляют йодированную соль, а среди школьников 13-18 - 8,1\%. В 1994-1995 гг. медианная КЙМ (мКЙМ) у лиц репродуктивного возраста г. Новосибирск составляла 47 мкг/л. В 2005 г. мКЙМ во взрослой популяции (45-69 лет) составила 107 мкг/л. В 2010 г. в подростковой популяции мКЙМ составила 93 мкг/л, КЙМ менее 50 мкг/л была определена в 7\% проб. В 2019 г. мКЙМ составила среди взрослых 19-25 лет 111 мкг/л (доля образцов мочи с КЙМ менее 50 мкг/л - 10,7\%), а у школьников 13-18 лет мКЙМ составила 123 мкг/л (доля образцов мочи с КЙМ менее 50 мкг/л - 14,5\%).

ЗАКЛЮчЕНИЕ. За 25-летний период наблюдения в г. Новосибирске отмечается значительное улучшение йодной обеспеченности населения. Зафиксирована низкая информированность молодого поколения жителей г. Новосибирска о йододефиците и мерах его профилактики.

КЛЮЧЕВЫЕ СЛОВА: дефицит йода; йодированная соль; йододефицитные заболевания.

\title{
EPIDEMIOLOGICAL STUDIES OF IODINE DEFICIENCY IN NOVOSIBIRSK: DATA OF 25-YEARS OBSERVATION
}

\author{
(c) Oksana D. Rymar, Svetlana V. Mustafina, Vlada I. Alferova*, Diana V. Denisova
}

Institution of Internal Medicine in Siberian Branch under the Russian Academy of Medical Sciences, Novosibirsk, Russia

BACKGROUND: According to the lodine Global Network, the Russian Federation is a region of mild iodine deficiency. At present, in Russia there is no systematic monitoring of the state of iodine deficiency in the regions. Existing legislation establishes the voluntary nature of iodine prophylaxis. In this regard, the issue of studying the iodine supply of the population of the Siberian region on the example of the city of Novosibirsk is relevant.

AIM: of research is to evaluate the availability of iodine and the effectiveness of the prevention of iodine deficiency among teenagers 13-18 years old and young people 19-25 years old in Novosibirsk.

MATERIALS AND METHODS: The research includes representative samples of pupils from 13 to 18 years old (612 people of both sexes, 62 of them have completed a questionnaire about the use of iodized salt, urine samples do determine UIC were taken from 60 of them ) and young people from 19 to 25 years old (101 people of both sexes. They all filled out the proposed questionnaire, 27 people were identified UIC). We used such methods as questionnaires, determination of UIC, comparison of the obtained data with the results of clinical and population studies conducted in Novosibirsk in 1994-1995, 2004-2005, 2009-2010.

RESULTS: In 2005 32.7\% of adult population consumed iodized salt. In 2010, 47\% of adolescence surveyed consumed iodized salt. According to a survey conducted in 2019 it was found that among adults 19-25 years old 19.8\% know that they 
consume iodized salt. Among schoolchildren from 13 to 18 years old, 8.1\% know for sure that use iodized salt. In 1994-1995 mUIC in persons of reproductive age in Novosibirsk was $47 \mu \mathrm{g} / \mathrm{L}$. In 2005 mUIC in the adult population (45-69 years old) was

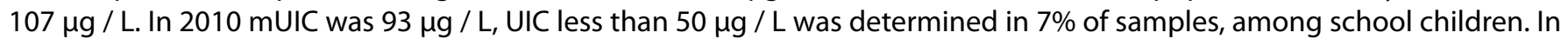
2019 MUIC amounted to $111 \mu \mathrm{g} / \mathrm{I}$ in adult population, the proportion of urine samples with UIC less than $50 \mu \mathrm{g} / \mathrm{I}-10.7 \%$. MUIC was $123 \mu \mathrm{g} / \mathrm{I}$ in 2019 among adolescence, the proportion of urine samples with UIC less than $50 \mu \mathrm{g} / \mathrm{I}-14.5 \%$.

CONCLUSIONS: Over the 24-year period of observation, there is a significant improving the iodine supply of the population in Novosibirsk. The low awareness of the young generation of the inhabitants of Novosibirsk about iodine deficiency and measures for its prevention was recorded.

KEYWORDS: iodine deficiency; iodized salt; iodine deficiency diseases (IDD).

По данным мировой статистики, дефицит йода одна из наиболее распространенных причин поражения головного мозга и нарушения психического развития, которую можно предотвратить [1, 2]. В настоящее время во всем мире двумя основными статистическими показателями, необходимыми для оценки статуса йодной обеспеченности, являются величина медианной концентрации йода в моче (мКЙМ) и доля образцов мочи с уровнем йода менее 50 мкг/л [1].

Согласно данным Глобальной сети по йоду (lodine Global Network), Российская Федерация является регионом умеренного дефицита йода, мКЙМ составляет 78 мкг/л. Эти данные были получены в результате ряда субнациональных исследований, проведенных в разные годы среди детей школьного возраста. Стоит отметить, что в отличие от других стран Восточной Европы и Центральной Азии в Российской Федерации не было проведено общенационального исследования обеспеченности населения йодом по стандартной методике [3].

Постановлением Главного государственного санитарного врача Российской Федерации от 03.04.1998 г. № 11 «О дополнительных мерах по профилактике йододефицитных состояний» установлен норматив содер-

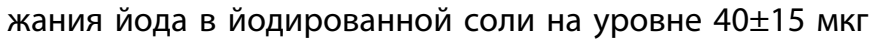
йода / г соли, а также сроки годности йодированной соли с использованием йодида калия - до 6 мес, с йодатом калия - до 9 мес.

В СССР еще в конце 1960-х гг. частота эндемического зоба, по официальным данным, снизилась до спорадического уровня, исчезли новые случаи кретинизма и зоба большого размера. Произошло это благодаря массовому использованию йодированной соли, производство которой к тому времени достигло миллиона тонн в год (более 4 кг на душу населения). Но затем, по мере нарастания экономических сложностей, производство йодированной соли в СССР в 1980-х гг. стало снижаться, а качество ее оставалось невысоким: более чем в половине образцов соли содержание йода было ниже предписанного стандартом [4].

С 1997 г. в магазинах Новосибирска регулярно имеется в продаже йодированная соль и другие продукты, обогащенные йодом. Сотрудниками НИИТПМ - филиал ИЦиГ СО РАН совместно с сотрудниками санитарно-гигиенической лаборатории ГУЗЦ ГСЭН №25 ФУ «Медбиоэкстрем» в 2008 г. была проведена оценка качества йодированной соли, имеющейся в продаже в Новосибирске. В представленных образцах содержание йода в соли соответствовало регламентирующему государственному стандарту. Это была йодированная соль следующих производителей: ОАО «Илецк-соль», Оренбургская обл., ОАО «Урал-калий», Пермская обл., Иркутская обл. Заларинский р-н п. Тыреть,
ЗАО «Валетек Продимпэкс», г. Москва [5]. В настоящее время в Российской Федерации отсутствует федеральный закон о всеобщем йодировании соли, а постановление Правительства Российской Федерации «О мерах по профилактике заболеваний, связанных с дефицитом йода» (от 05.10.1999 № 1119) устанавливает добровольный порядок профилактики йододефицитных заболеваний (ЙДЗ) путем использования йодированной соли и других обогащенных йодом продуктов.

Таким образом, личный выбор поваренной соли, используемой для приготовления пищи в семье, продуктов питания или конкретных средств профилактики ЙДЗ определяет степень защищенности каждого конкретного человека от последствий йодного дефицита и в целом приводит к результатам на уровне популяции. Насколько этот выбор будет осознанным, зависит от большого числа факторов, при этом повышение информированности населения о проблеме ЙДЗ и средствах их профилактики не является гарантией принятия верного решения.

\section{ЦЕЛЬ}

Оценить обеспеченность йодом и эффективность мер по профилактике йодного дефицита среди подростков 13-18 лет и лиц молодого возраста 19-25 лет г. Новосибирска.

\section{МАТЕРИАЛЫ И МЕТОДЫ}

\section{Дизайн исследования}

Дизайн исследования - одномоментное (кросс-секционное) популяционное исследование случайной репрезентативной выборки школьников 13-18 лет и молодых людей 19-25 лет обоего пола.

На первом этапе было проведено одномоментное исследование, включающее молодых людей в возрасте 19-25 лет и школьников 13-18 лет.

На втором этапе осуществлялось сравнение показателей, полученных в 2019 г., с данными аналогичных исследований, проводимых в г. Новосибирске в период с 1995 по 2015 гг.

\section{Анализ в подгруппах}

Для оценки динамики йодной обеспеченности на первом этапе исследования были сформированы две группы участников: группа школьников, состоявшая из 62 детей обоего пола в возрасте 13-18 лет, и группа взрослых, включавшая 101 человека обоего пола в возрасте 19-25 лет. Полученные данные сопоставлялись с результатами клинико-популяционных исследований, 
проводимых на территории г. Новосибирска в 1994-1995, 2004-2005, 2009-2010 гг. В рамках международных проектов MONICA (1994-1995 гг.), HAPIEE (2004-2005 гг.) и одномоментного кросс-секционного популяционного скрининга случайной репрезентативной выборки школьников 14-17 лет (2009-2010 гг.) проводилось дополнительное изучение структурно-функционального состояния щитовидной железы (ЩЖ) и йододефицита в популяции взрослых и подростков г. Новосибирска. В 1994-1995 гг. было обследовано 200 мужчин и женщин репродуктивного возраста (25-34 лет), в 2004-2005 гг. 280 человек обоего пола 45-64 лет и в 2009-2010 гг. 215 школьников обоего пола (14-17 лет).

В 2019 г. был проведен скрининг подростков - учащихся средних общеобразовательных школ. Из 20 школ одного из районов Новосибирска методом случайных чисел было отобрано 6 школ, в которых проводилось сплошное обследование учащихся 8-11 классов. Всего обследовано 612 человек (41,2\% мальчиков, 58,8\% девочек). Из выборки подростков случайным образом были отобраны 62 человека обоего пола, которым было проведено анкетирование, включавшее заполнение формализованного опросника об использовании йодированной соли. У 60 из них были взяты образцы утренней мочи для определения уровня экскреции йода с мочой.

Также в 2019 г. на основе скрининга подростков, проводившегося ранее в 2014 г., была сформирована когорта лиц 19-25 лет, с целью оценки у них пятилетней количественной динамики (2014-2019 гг.) конвенционных факторов риска сердечно-сосудистых заболеваний (уровни АД, общего холестерина сыворотки крови и его фракций, уровень глюкозы сыворотки крови, индекса массы тела). Обследование начато в 2019 г., на момент проведения нашего исследования был обследован 101 человек обоего пола, все они заполнили предлагаемую анкету. У каждого четвертого из участников были взяты образцы утренней мочи для определения уровня экскреции йода с мочой.

\section{Методы}

У всех участников осуществлялся забор утренней мочи для определения уровня экскреции йода с мочой. В специально отведенных кабинках в утренние часы проводился сбор образцов мочи в маркированные одноразовые стаканы, после чего мочу с помощью одноразовых пластиковых пипеток переливали в пробирки с плотно закручивающимися крышками и помечали наклейкой с индивидуальным номером обследуемого.

Определение концентрации йода в моче в разовых порциях в 1994-1995 и 2005 гг. проводили с использованием церий-арсенитного метода. Эти исследования были выполнены в лаборатории клинической биохимии Эндокринологического научного центра РАМН г. Москвы (руководитель лаборатории - д.м.н. А.В. Ильин). В 2010 г. определение концентрации йода в разовых утренних порциях мочи у школьников Новосибирска проведено с использованием инверсионно-вольтамперометрического метода согласно методическим рекомендациям МУ 08-47/148 (по реестру аккредитованной метрологической службы). Метод аттестован в соответствии с ГОСТ Р 8.563-96 и внесен в Государственный реестр методов метрологического контроля и надзора ФР.1.31.2004.01109. Анализ производили с использованием аналитического вольтамперометрического комплекса СТА (Томск, Политехнический университет НПФ «ЮМХ»). Исследования проводились в научно-исследовательской лаборатории общей патологии и функциональной морфологии Кемеровской Государственной медицинской академии (зав. лаб. - к.м.н. Зинчук В.Г.).

Определение КЙМ в 2019 г. проводилось на базе лаборатории ИНВИТРО (заведующий клинико-диагностической лабораторией «ИНВИТРО-Новосибирск» врач клинической лабораторной диагностики В.В. Андрушкевич) методом масс-спектрометрии с индуктивно связанной аргоновой плазмой (ИСП-МС).

Результаты содержания йода в моче представлены в виде абсолютных значений концентраций - мкг/л. В связи с очень вариабельным уровнем йода в образцах мочи оценивали медиану, а не средние значения [1]. Для оценки тяжести йодной недостаточности нами был использован показатель мКЙМ. Популяционный показатель мКЙМ менее 20 мкг/л оценивается как тяжелый йододефицит; от 20 до 50 мкг/л - йододефицит средней степени; от 50 до 99 мкг/л - йододефицит легкой степени; 100-299 мкг/л - отсутствие йододефицита; 300 и более мкг/л - избыточное потребление йода [1].

\section{Этическая экспертиза}

Для включения в обследование обязательным условием служило получение письменного информированного согласия (от взрослых, от обследуемого подростка и его родителей). Все исследования были одобрены локальным этическим комитетом НИИТПМ филиала ИЦиГ СО РАН (протоколы №29 от 26.03.2019 г. и №58 от 23.04.2019 г.).

\section{Статистический анализ}

Статистическая обработка полученных результатов выполнена с помощью пакета SPSS (V. 13.0) и включала создание базы данных, автоматизированную проверку качества подготовки информации и статистический анализ. Оценку средних значений количественных показателей проводили с помощью процедуры однофакторного дисперсионного анализа, определения мКЙМ. Использовали стандартные критерии оценки статистических гипотез: t-критерий Стьюдента в случае нормального распределения количественных показателей. Проверку гипотез проводили для уровня вероятности 95\% $(\mathrm{p}<0,05)$.

\section{РЕЗУЛЬТАТЬ}

\section{Объекты (участники) исследования}

Для оценки уровня йодной обеспеченности были сформированы две группы участников исследования. Первая группа была набрана на базе скрининга состояния здоровья молодого населения (19-25 лет) г. Новосибирска в 2019 г. (n=101 человек, 67 девушек, 34 юноши), из обследованной группы была отобрана каждая четвертая проба - 27 человек для определения КЙМ.

Группа подростков набиралась в рамках скрининга состояния здоровья школьников (13-18 лет) Октябрьского района г. Новосибирска, проводимого в 2019 г. В выборку были включены 62 человека (39 девушек, 23 юношей), у 60 из них были взяты образцы мочи для определения КЙМ. 


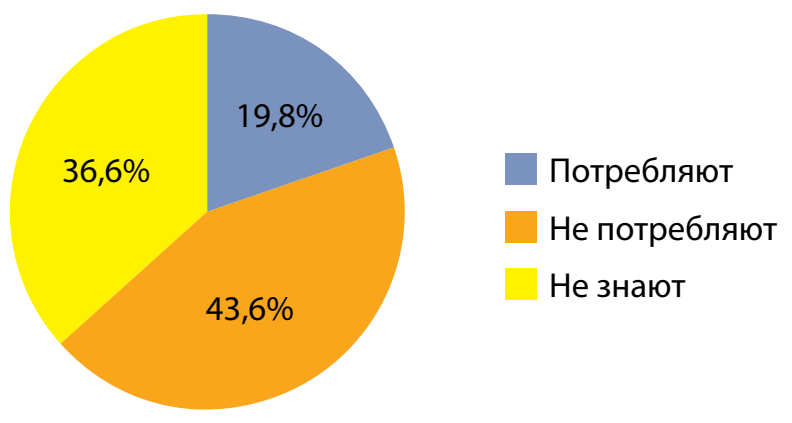

Рисунок 1. Потребление йодированной соли среди населения 19-25 лет г. Новосибирска в 2019 г. по данным опроса.

Наряду с полевыми исследованиями на втором этапе был проведен сравнительный анализ полученных данных с показателями аналогичных исследований, проводимых в г. Новосибирске в 1995-2015 гг.

\section{Основные результаты исследования}

В ходе исследований, проведенных нами в 2019 г., были получены следующие данные. По данным анкетирования, среди взрослых 19-25 лет только 19,8\% знают о том, что потребляют йодированную соль, из них $17,9 \%$ девушек и 23,5\% юношей, р>0,05. Остальная часть опрошенных утверждают, что не употребляют йодированную соль (43,6\%), либо не знают, какая соль используется в их семье (36,6\%) (рис. 1$)$.

Полученные результаты свидетельствуют о низкой осведомленности опрошенных лиц о виде соли, используемой в их семьях. Прием йодсодержащих лекарственных препаратов (йодомарина) в анамнезе отмечен у 16,8\% опрошенных (23,9\% девушек и 2,9\% юношей, $\mathrm{p}=0,009)$. По полученным нами данным, мКЙМ в группе

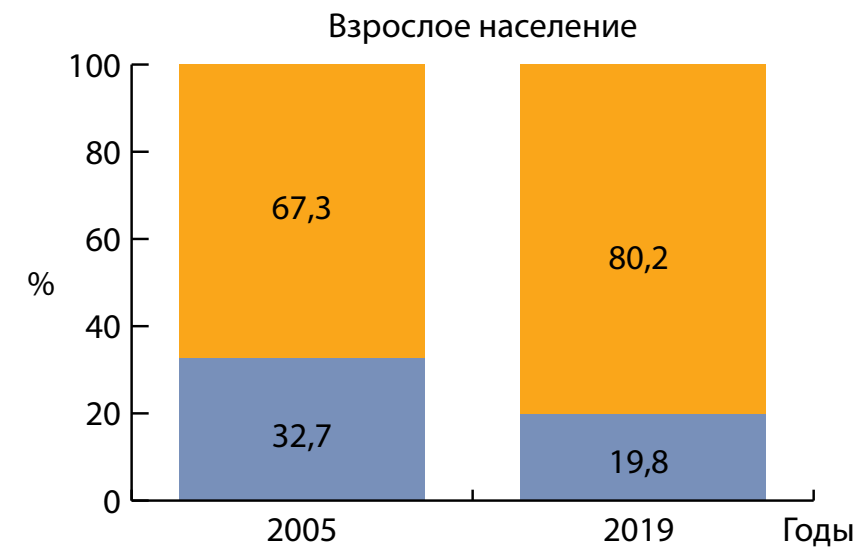

Потребляют — Не потребляют / Не знают

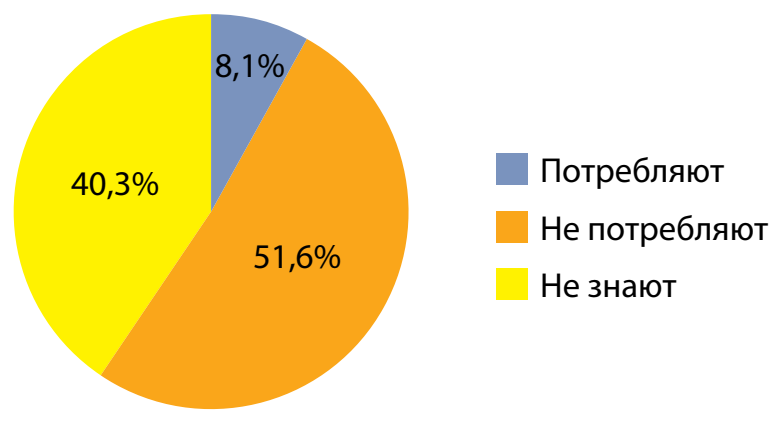

Рисунок 2. Потребление йодированной соли среди подростков г. Новосибирска в 2019 г. по данным опроса.

молодых людей 19-25 лет составила 111 мкг/л, причем доля образцов мочи с КЙМ менее 50 мкг/л - 10,7\%.

Среди школьников 13-18 лет, согласно данным анкетирования, всего $8,1 \%$ точно знают, что употребляют йодированную соль (10,3\% девочек и 4,3\% мальчиков, р>0,05). При этом 51,6\% респондентов отрицают использование йодированной соли, а 40,3\% не знают, какую соль используют в их семье для приготовления пищи (рис. 2).

Значительная часть школьников - 40,3\% недостаточно информированы о том, какая соль используется в их семьях для приготовления пищи. Принимают йодсодержащие лекарственные препараты (йодомарин) $21 \%$ опрошенных (20,5\% девочек и 21,7\% мальчиков, p=0,009). МКЙМвгруппе школьников составила 123 мкг/л, а доля образцов мочи с КЙМ менее 50 мкг/л - 14,5\%.

При сравнении результатов исследования, проведенного нами в 2019 г., с данными прошлых лет, обращают на себя внимание, что информированность о потреблении йодированной соли за 25 лет наблюдения снизилась как в группе взрослых, так и в группе школьников (рис. 3,4$)$.

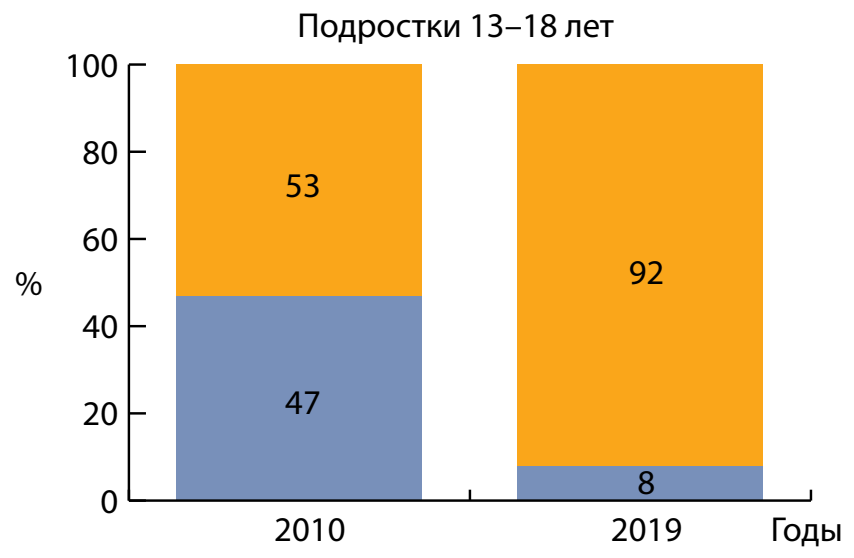

Потребляют Не потребляют / Не знают

Рисунок 3. Результаты опроса взрослого и подросткового населения г. Новосибирска об использовании йодированной соли в их семьях.

$\%$

32,7

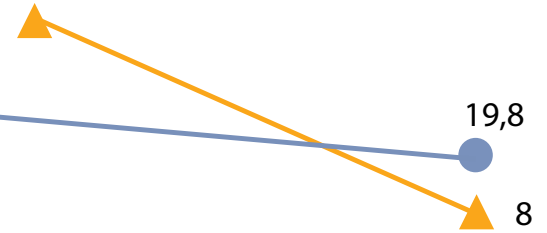

2010
2019

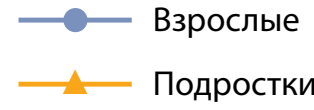

\section{5}

Рисунок 4. Динамика потребления йодированной соли населением г. Новосибирска (2005-2019 гг.) (по данным анкетирования) 


\section{Нежелательные явления}

В процессе проведения исследования нежелательные явления не ожидались и не были отмечены.

\section{ОБСУЖДЕНИЕ}

\section{Резюме основного результата исследования}

С 1995 г. отмечается рост показателей мКЙМ. Вместе с тем, по данным опроса, зафиксирована низкая информированность молодого населения г. Новосибирска о мерах профилактики дефицита йода путем потребления йодированной соли.

\section{Обсуждение основного результата исследования}

Исторически Новосибирская область и г. Новосибирск не являлись очагами зобной эндемии. Однако когда в середине 1980-х гг. в Новосибирск, как и во многие города России, прекратились поставки йодированной соли, ситуация с йодной обеспеченностью начала стремительно меняться. Прекращение проведения массовой йодной профилактики привело к тому, что население Новосибирска и других сибирских регионов стало испытывать дефицит йода в питании [5]. По результатам популяционного исследования, проведенного сотрудниками НИИТП - филиала ИЦиГ СО РАН в 1994-1995 гг., мКЙМ у лиц репродуктивного возраста г. Новосибирска составляла 47 мкг/л, у 83\% жителей молодого возраста (25-34 лет) КЙМ в пробах была менее 100 мкг/л [6, 7].

В ходе исследования, проведенного на базе НИИТПМ - филиала ИЦиГ СО РАН в 2005 г., было осмотрено и проанкетировано 560 человек (45-69 лет): 275 (49,1\%) женщин и 285 (50,9\%) мужчин. Среди опрошенных потребляли йодированную соль 32,7\%, из них женщин 42,5\% и мужчин - 23,2\%. В 30\% случаев мужчины не знали, какую соль покупает супруга. МКЙМ во взрослой популяции составила 107 мкг/л. Среди образцов мочи в 13\% проб показатели КЙМ оказались менее 50 мкг/л [5].

Показатели мКЙМ, определенные у взрослых, сравнимы в 2005 г. и в 2019 г.: 107 мкг/л и 111 мкг/л соответ-

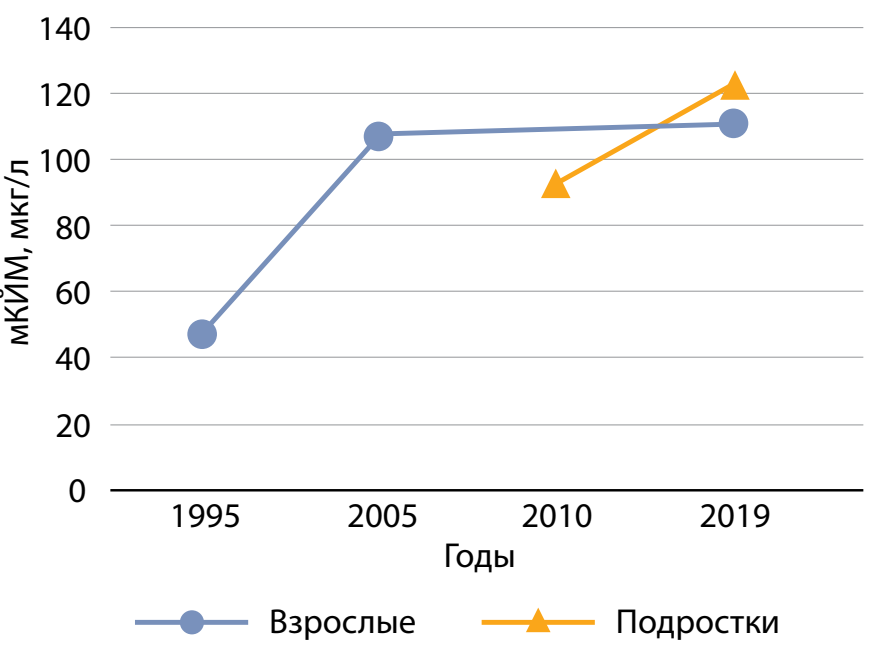

Рисунок 5. Динамика медианы концентрации йода в моче (мкг/л) у жителей Новосибирска по результатам мониторинга йододефицитных заболеваний с 1995 по 2019 гг.

ственно; у подростков - 93 мкг/л в 2010 г. и 123 мкг/л в 2019 г. (рис. 5). У обследованных взрослых количество образцов с КЙМ <50 мкг/л сопоставимо в динамике: в 2005 г. - 13\% и в 2019 г. - 10,7\%; у подростков возросло с $7 \%$ до $14,5 \%, \mathrm{p}<0,05$.

При сравнении между собой результатов обследования обеих групп обращает на себя внимание схожесть процентного распределения проб мочи с нормальной, сниженной и повышенной КЙМ (рис. 6).

Таким образом, по полученным нами данным, дефицит йода не выявлен ни в группе взрослых лиц 19-25 лет, ни в группе школьников 13-18 лет, обследованных в 2019 г. Показатель мКЙМ во взрослой популяции с 1995 г. увеличился более чем в 2 раза, а в популяции школьников за период с 2010 по 2019 гг. мКЙМ увеличилась на $32,3 \%$.

В 2009-2010 гг. с целью оценки потребления йодированной соли были проанкетированы подростки (14-17 лет), из них мальчиков 47\%, девочек 53\%. 47\% опрошенных потребляли йодированную соль, при этом 53\% проанкетированных подростков ответили, что

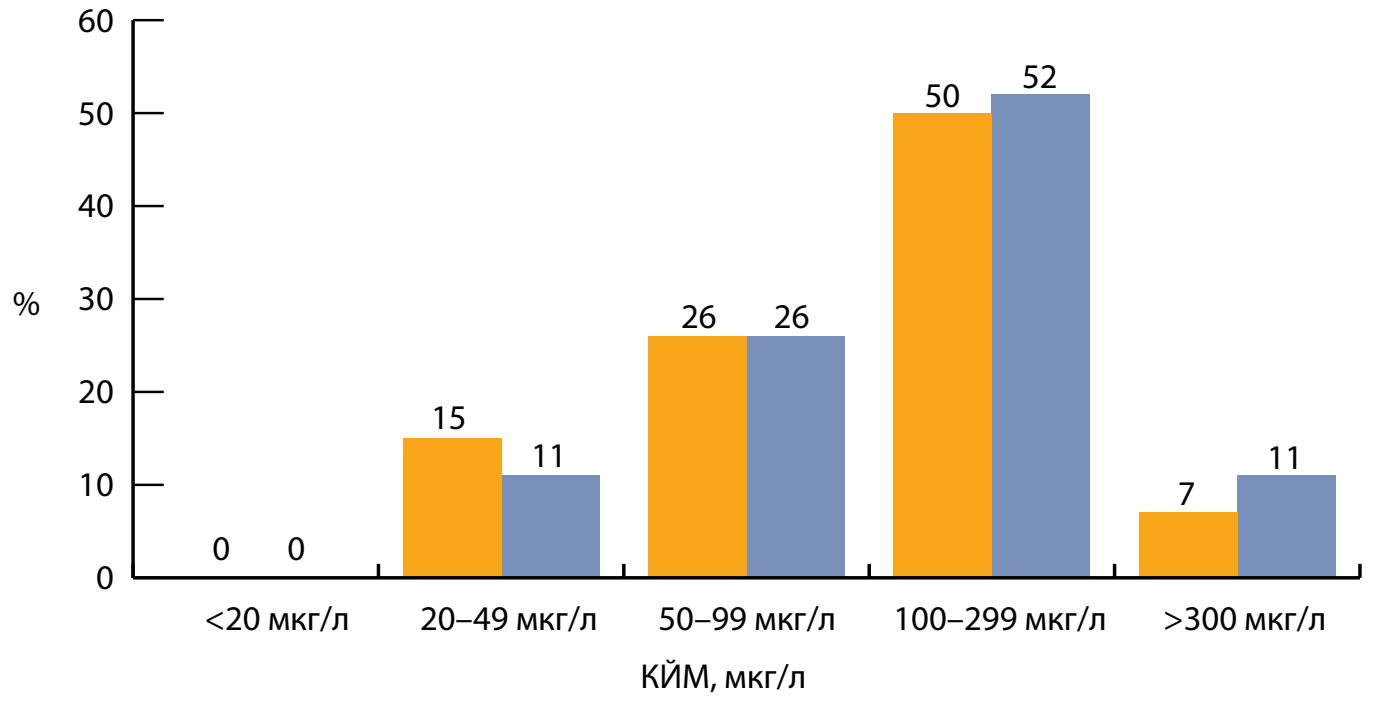

Школьники 13-18 лет — Взрослые 19-25 лет

Рисунок 6. Процентное соотношение проб мочи с нормальной, сниженной и повышенной КЙМ (2019). 
не употребляют йодированную соль или не знают, какую соль используют дома для приготовления пищи. В той же репрезентативной выборке подростков была определена экскреция йода с мочой. МКЙМ у подростков составила 93 мкг/л. Среди образцов мочи показатели КЙМ менее 50 мкг/л были определены в 7\% проб [7].

Согласно рекомендациям ВО3, эффективной массовая йодная профилактика признается только в том случае, когда более 90\% всех домохозяйств (семей) используют в быту йодированную соль [8]. В большинстве регионов мира йодированная соль является основным источником йода в рационе человека и, как следствие, основным средством массовой йодной профилактики. Установлено, что более $80 \%$ йода выводится из организма почками, поэтому КЙМ достаточно точно отражает величину его потребления с пищей. КЙМ является количественным, прямым показателем йодной обеспеченности. Вместе с тем, из-за высоких индивидуальных колебаний уровня йода в моче, этот метод нельзя использовать для оценки величины потребления йода у отдельного человека. На популяционном же уровне этот метод отражает величину потребления йода населением обследуемого региона $[1,9]$.

Нами получены данные о низком уровне потребления йодированной соли, но нормальных показателях мКЙМ, вероятно, расхождения показателей потребления йодированной соли и мКЙМ отчасти обусловлены тем, что многие подростки и молодые люди, проживающие с родителями, не проявляют интереса к тому, какую соль используют в их семье для приготовления пищи. Возможно, часть респондентов, ответивших на вопрос об употреблении йодированной соли «не знаю», на самом деле ее употребляют, с этим и могут быть связаны нормальные показатели мКЙМ. Тем не менее мКЙМ в г. Новосибирске на протяжении 25 лет наблюдения продолжает возрастать (рис. 5).

В соответствии с постановлением Главного государственного санитарного врача Российской Федерации от 23.07.2008 (ред. от 25.03.2019) «Об утверждении СанПиН 2.4.5.2409-08» (вместе с «СанПиН 2.4.5.2409-08. Санитарно-эпидемиологические требования к организации питания обучающихся в общеобразовательных учреждениях, учреждениях начального и среднего профессионального образования. Санитарно-эпидемиологические правила и нормативы») устанавливается обязательное использование йодированной соли при приготовлении блюд и кулинарных изделий поваренной пищевой йодированной соли с 01.01.2020. Также Министерством здравоохранения Российской Федерации подготовлена инициатива о профилактике заболеваний, связанных с недостатком йода. Согласно проекту Федерального закона «О профилактике заболеваний, вызванных дефицитом йода», размещенного на федеральном портале проектов нормативных актов, в Российской Федерации будет обязательным использование йодированной соли при изготовлении любых хлебобулочных изделий, начиная с 01 июня 2020 г. А с 1 июня 2021 г. в России станет обязательным обогащение йодом пищевой соли сортов экстра, высшего и первого, помолов N0 и N1, за исключением отдельных видов пищевой соли указанных сортов и помолов, перечень которых устанавливается уполномоченным федеральным органом исполни- тельной власти. Помимо этого, начиная с 1 июня 2022 г. все пищевые продукты на территории Российской Федерации должны будут производиться с использованием только йодированной соли. В тех случаях, когда технологический процесс не будет допускать использование йодированной соли, производители обязаны будут обогащать йодом продукты иными возможными способами [10]. Данный законопроект на момент написания статьи готовится к передаче на рассмотрение в Правительство Российской Федерации.

Что касается использования йодированной соли в домохозяйствах (семьях), то этот вопрос по-прежнему остается в нашей стране сугубо индивидуальным и зависит только от степени ответственности граждан как за свое здоровье, так и за здоровье детей.

В Кемеровской области в 2008 г. в популяции школьников 7-17 лет г. Междуреченска наблюдался дефицит йода легкой степени тяжести (мКЙМ составила 90 мкг/л). Доля образцов мочи с уровнем экскреции йода менее 50 мкг/л составила 11,4\% [11]. По данным повторного исследования, в 2016 г. нормальные показатели КЙМ были выявлены у 46,4\% школьников, при этом доля образцов с уровнем КЙМ менее 50 мкг/л составила всего 1,3\% [12]. Таким образом, на территории г. Междуреченска в 2016 г. отмечалась положительная динамика йодной обеспеченности, хотя дефицит йода на данной территории все еще не ликвидирован.

В Тюменской области в 2012 г. мКЙМ среди школьников 8-12 лет составляла 106,9 мкг/л, что по критериям ВО3 соответствует достаточному потреблению йода. При этом полученные значения оказались ниже, чем в аналогичном исследовании, проведенном в 2000-2003, 2009 гг. (мКЙМ в данных исследованиях составляла 125 мкг/л и 142 мкг/л соответственно). Авторы исследования объясняют подобную динамику добровольным характером профилактики йододефицитных заболеваний [13, 14]. Важно отметить, что в Тюменской области с 1997 г. реализуется региональная программа профилактики йодного дефицита в соответствии с постановлением Главного государственного санитарного врача по Тюменской области от 15.09.1997 № 17 «О профилактике йододефицитных состояний» и распоряжением губернатора Тюменской области от 30.10.1997 № 694-р «О профилактике йододефицитных состояний» [13]. Эти меры были связаны с тем, что по результатам первичных эпидемиологических исследований 1994-1996 гг. на территории Тюменской области был выявлен природно-обусловленный йодный дефицит [15].

Коллектив авторов из Томска в 2016 г. опубликовал результаты исследования, в ходе которого оценивали динамику йодного дефицита в г. Томске с 1998 по 2014 гг. МКЙМ за этот период увеличилась с 73,1 мкг/л в 1998 г. до 94 мкг/л в 2014 г. (число обследованных школьников составило 264 и 362 соответственно). Таким образом, на территории Томской области сохраняется йодный дефицит легкой степени тяжести, что требует продолжения проводимых мероприятий, направленных на устранение дефицита йода в данном регионе [16].

В период с 19 ноября по 3 декабря 2019 г. в г. Москве был проведен опрос интернет-пользователей по поводу йодного дефицита и методах его профилактики, среди прочих был в предлагаемой анкете и вопрос 
об использовании йодированной соли в семьях респондентов. Всего в опросе приняли участие 9309 человек со всей России. Результаты опроса показали, что 60,4\% опрошенных используют в быту йодированную соль, только половина $(52,73 \%)$ респондентов, проживающих на территории Западной Сибири, потребляют йодированную соль. Однако очень небольшое количество (57 человек) жителей Западной Сибири приняли участие в опросе [17].

Таким образом, 25-летние данные по оценке йодного статуса жителей г. Новосибирска отображают положительную тенденцию по уменьшению йодного дефицита. Однако массовая йодная профилактика недостаточно эффективна как в г. Новосибирске, так и по всей стране. Полученные данные по опросу интернет-пользователей и представленные в нашей работе результаты свидетельствуют о низком уровне информированности населения РФ о значимости йодной профилактики и о необходимости принятия федерального закона «О профилактике заболеваний, вызванных дефицитом йода».

\section{Ограничения исследования}

Не была определена концентрация йода в образцах соли, используемой в семьях участников исследования.

В исследованиях, проводимых в 1994-95, 2005, 2010 и 2019 гг., определение КЙМ проводилось разными методами (церий-арсенитным, инверсионно-вольтамперометрическим и методом ИСП-МС).

\section{ЗАКЛЮЧЕНИЕ}

По полученным нами данным, за 25-летний период наблюдения с 1994-1995 по 2019 гг. в г. Новосибирске отмечается значительное улучшение йодной обеспеченности населения, что отражено в нормализации и увеличении показателя мКЙМ среди молодых лиц репродуктивного возраста 19-25 лет и школьников 13-18 лет.
При этом результаты опроса населения показали очень низкую информированность молодого поколения жителей г. Новосибирска о йододефиците и мерах его профилактики. Также нами отмечена следующая тенденция: чем моложе возраст опрашиваемых, тем меньше значимости они придают проблеме йодного дефицита, что видно по данным опроса.

При разработке стратегии профилактики дефицита йода необходимо уделять внимание информированию граждан о важности устранения йододефицита, а также целенаправленно формировать мотивацию особенно у молодых граждан к потреблению йодированной соли.

\section{ДОПОЛНИТЕЛЬНАЯ ИНФОРМАЦИЯ}

Источник финансирования. Статья подготовлена в рамках бюджетных тем НИИТПМ - филиал ИЦиГ СО РАН ГЗ № 0324-2018-0001, Рег. № AАAА-А17-117112850280-2, ГЗ № 0259-2019-0006, Рег. № ААAА-А19-119101490005-56, РФФИ 19-013-00800.

Конфликт интересов. Авторы декларируют отсутствие явных и потенциальных конфликтов, связанных с публикацией данной статьи.

Участие авторов. Рымар О.Д. - концепция и дизайн работы, финальное редактирование статьи; Мустафина С.В. - сбор материала, дизайн поисково-аналитической работы, написание текста статьи; Алфёрова В.И. - сбор, обработка и анализ материала, написание текста статьи; Денисова Д.В. - организация скрининга подростков и молодых людей, написание раздела «Материаы и методы».

Все авторы внесли значимый вклад в проведение поисково-аналитической работы и подготовку статьи, прочли и одобрили финальную версию до публикации.

Благодарность: Авторы статьи выражают благодарность член.корр. СО РАН, д.м.н. Рагино Ю.И. за поддержку и возможность получить данные на скринингах 2019 г., главным исследователям проекта НAPIEE акад. СО РАН, д.м.н. Никитину Ю.П, профессору, д.м.н. Малютиной С.К. за помощь в проведении скрининга населения в 2005 и 1994-1995 гг., а также всем сотрудникам, работающим на скрининге, за помощь в сборе материала.

\section{СПИСОК ЛИТЕРАТУРЫ | REFERENCES}

1. Детский фонд ООН (ЮНИСЕФ), Глобальная сеть по йоду (IGN). Рекомендации по мониторингу программ йодирования соли и оценке статуса йодной обеспеченности населения (русскоязычная версия) // Клиническая и экспериментальная тиреоидология. 2018. — T.14. — №2. — C. 100-112. [United Nations Children's Fund (UNICEF), lodine Global Network (IGN). Guidance on the monitoring of salt iodization programmes and determination of population iodine status: Russian language version. Clinical and experimental thyroidology. 2018;14(2):100-112. (In Russ.)]. doi: 10.14341/ket9734.

2. Суханов А.В., Рымар О.Д., Мустафина С.В., Денисова Д.В. Показатели когнитивной функции у подростков, проживающих в регионе с легким йододефицитом // Мир науки, культуры, образования. - 2013. - №1. - C. 267-271. [Sukhanov AV, Rymar OD, Mustafina SV, Denisova DV. Adolescent's cognitive functions condition in region with mild iodine deficiency. Mir nauki, kul'tury, obrazovania. 2013;(1):267-271. (In Russ.)].

3. Алферова В.И., Мустафина С.В., Рымар О.Д. Йодная обеспеченность в России и мире: что мы имеем на 2019 год? // Клиническая и экспериментальная тиреоидология. - 2019. T.15. - №2. - C. 73-82. [Alferova VI, Mustafina SV, Rymar OD. lodine status of the population in Russia and the world: what do we have for 2019? Clinical and experimental thyroidology. 2019;15(2):73-82. (In Russ.)]. doi: 10.14341/ket10353.

4. Герасимов Г.А. Квадратура круга // Клиническая и экспериментальная тиреоидология. — 2016. - Т.12. №3. - C. 6-11. [Gerasimov GA. Quadrature of the circle. Clinical and experimental thyroidology. 2016;12(3):6-11. (In Russ.)]. doi: 10.14341/ket201636-11.

5. Никитин Ю.П., Рымар О.Д., Мустафина С.В., и др. Обеспеченность йодом взрослого населения Новосибирска // Вопросы питания. 2008. — T.77. — №2. - C. 64-66. [Nikitin YuP, Rymar OD, Mustafina SV, et al. Providing of adult population of Novosibirsk with iodine. Problems of nutrition. 2008;77(2):64-66. (In Russ.)].

6. Рымар О.Д. Распространенность патологии шитовидной железы и связь с некоторыми факторами риска хронических неинфекционных заболеваний у жителей молодого возраста г. Новосибирска: Автореф. дисс. ... канд. мед. наук. Новосибирск, 1999. — 22 с. [Rymar OD. Rasprostranennost' patologii shchitovidnoi zhelezy i svyaz's nekotorymi faktorami riska khronicheskikh neinfektsionnykh zabolevanii u zhitelei molodogo vozrasta g. Novosibirska. [dissertation abstract] Novosibirsk; 1999. 22 р. (In Russ.)]. Доступно по: Ссылка доступна на 12.03.2020.

7. Рымар О.Д., Мустафина С.В., Симонова Г.И., и др. Эпидемиологические исследования йодного дефицита и тиреоидной патологии в крупном центре Западной Сибири в 1995-2010 гг. (на примере Новосибирска) // Клиническая и экспериментальная тиреоидология. - 2012. - Т.8. №2. - C. 50-54. [Rymar OD, Mustafina SV, Simonova Gl, et al. Epidemiological evaluation of iodine deficiency and thyroid disorders in the megalopolis of Western Siberia in 1995-2010. Clinical and experimental thyroidology. 2012;8(2):50-54. (In Russ.)]. doi: 10.14341/ket20128250-54. 
8. Zimmermann MB. Symposium on geographical and geological influences on nutrition': iodine deficiency in industrialised countries. Proc Nutr Soc. 2010;69(1):133-143. doi: 10.1017/S0029665109991819.

9. Методические указания 2.3.7.1064-01. Контроль программы профилактики йоддефицитных заболеваний путем всеобщего йодирования соли. - М.; 2001. [Methodological recommendations 2.3.7.1064-01. Kontrol' programmy profilaktiki ioddefitsitnykh zabolevanii putem vseobshchego iodirovaniya soli. Moscow; 2001. (In Russ.)].

10. Проект Федерального закона «О профилактике заболеваний, вызванных дефицитом йода». [Project of Federal Law «O profilaktike zabolevanii, vyzvannykh defitsitom ioda». (In Russ.)]. Доступно по: https://regulation.gov.ru/projects\#npa=89946. Ссылка доступна на 12.03.2020.

11. Тапешкина Н.В. Гигиеническая оченка питания детей школьного возраста и пути ее оптимизачии (на примере юга Кузбасса): Автореф. дисс. ... канд. мед. наук. - Пермь; 2009. [Tapeshkina NV. Gigienicheskaya otsenka pitaniya detei shkol'nogo vozrasta i puti ee optimizatsii (na primere yuga Kuzbassa). [dissertation abstract] Perm; 2009. (In Russ.)]. Доступно по: https://search.rsl.ru/ru/record/01004663735. Ссылка доступна на 12.03.2020

12. Тапешкина Н.В., Перевалов А.Я. Оценка эффективности профилактики йодного дефицита среди детского населения города Междуреченска Кемеровской области // Гигиена и санитария. - 2016. - Т.95. — №5. - C. 471-476. [Tapeshkina NV, Perevalov AYa. Assessment of the efficiency of prevention of iodine deficiency among the children's population of the city of Mezhdurechensk of the Kemerovo region. Hygiene and sanitation. 2016;95(5):471-476. (In Russ.)]. doi: 10.18821/0016-9900-2016-95-5-471-476.

13. Суплотова Л.А., Макарова О.Б., Ковальжина Л.С., Шарухо Г.В. Профилактика йодного дефицита в Тюменской области: успех или неудача? // Клиническая и экспериментальная миреоидология. - 2015. - Т.11. - №3. - C. 39-46. [Suplotova LA, Makarova OB, Kovalzhina LS, Sharuho GV. Prevention of iodine deficiency in the Tyumen region: success or failure? Clinical and experimental thyroidology. 2015;11(3):39-46. (In Russ.)] doi: 10.14341/ket2015339-46

14. Герасимов Г.А. Россия - страна контрастов // Клиническая и экспериментальная тиреоидология. - 2017. - Т.13. №2. - C. 6-12. [Gerasimov GA. Russia - the land of contrasts. Clinical and experimental thyroidology. 2017;13(2):6-12. (In Russ.)]. doi: 10.14341/ket201726-12.

15. Суплотова Л.А. Эпидемиология йоддефииитных заболеваний в различных климатогеографических районах Западной Сибири: Автореф. дис. ... докт. мед. наук. - Тюмень, 1997. 38 c. [Suplotova LA. Epidemiologiya ioddefitsitnykh zabolevanii $\checkmark$ razlichnykh klimatogeograficheskikh raionakh Zapadnoi Sibiri. [dissertation abstract] Tyumen; 1997. 38 р. (In Russ.)]. Доступно по: https://search.rsl.ru/ru/record/01000761362. Ссылка доступна на 12.03.2020.

16. Олейник О.А., Самойлова Ю.Г., Юрченко Е.В., Зинчук С.Ф. Динамика эпидемиологических критериев йодного дефицита В г. Томске за 15 лет. В сб.: Тезисы VII Всероссийского конгресса эндокринологов «Достижения персонализированной медицины сегодня - результат практического здравоохранения завтра». - М.: УП Принт, 2016. - С. 8. [Oleinik OA, Samoilova YuG, Yurchenko EV, Zinchuk SF. Dinamika epidemiologicheskikh kriteriev iodnogo defitsita $\vee$ g. Tomske za 15 let. In: (Collection of theses) Tezisy VII Vserossiiskogo kongressa endokrinologov «Dostizheniya personalizirovannoi meditsiny segodnya — rezul'tat prakticheskogo zdravookhraneniya zavtra». Moscow: UP Print; 2016. P. 8. (In Russ.)].

17. Терехов П.А., Рыбакова А.А., Терехова М.А., Трошина Е.А. Информированность населения Российской Федерации о йодном дефиците, его влиянии и способах профилактики йододефицитных заболеваний // Клиническая и экспериментальная тиреоидология. - 2019. - Т.15. №3. - C. 118-123. [Terekhov PA, Rybakova AA, Terekhova MA, Troshina EA. Awareness of the population in Russian Federation about iodine deficiency, its effects and methods for prevention of iodine deficiency disorders. Clinical and experimental thyroidology. 2019;15(3):118-123. (In Russ.)]. doi: 10.14341/ket12239.

\section{ИНФОРМАЦИЯ ОБ АВТОРАХ [AUTHORS INFO]}

*Алфёрова Влада Игоревна [Vlada I. Alferova]; адрес: Россия, 630089, г. Новосибирск, ул. Бориса Богаткова, д. 175/1 [address: 175/1, Borisa Bogatkova street, 630089 Novosibirsk, Russian Federation]; ORCID: https://orcid.org/0000-0002-1645-5523; eLibrary SPIN: 1129-0599; e-mail: lady.alfyorova2009@yandex.ru

Рымар Оксана Дмитриевна, д.м.н. [Oksana D. Rymar, MD, PhD]; ORCID: https://orcid.org/0000-0003-4095-0169; eLibrary SPIN: 8345-9365; e-mail: orymar23@gmail.com

Мустафина Светлана Владимировна, д.М.H. [Svetlana V. Mustafina, MD, PhD];

ORCID: https://orcid.org/0000-0003-4716-876X; eLibrary SPIN: 8395-1395; e-mail: svetlana3548@gmail.com

Денисова Диана Вахтанговна, д.м.н. [Diana V. Denisova, MD, PhD]; e-mail: denisovadiana@gmail.com; ORCID: https://orcid.org/0000-0002-2470-2133; SPIN 9829-7692

\section{КАК ЦИТИРОВАТЬ}

Рымар О.Д., Мустафина С.В., Алфёрова В.И., Денисова Д.В. Эпидемиологические исследования йодного дефицита В г. Новосибирске: данные 25-летнего наблюдения // Клиническая и экспериментальная тиреоидология. - 2020. T. 16. — №2. - C. 4-11. doi: https://doi.org/10.14341/ket12539

Получена: 20.07.2020. Одобрена: 24.08.2020. Опубликована online: 07.10.2020

\section{TO CITE THIS ARTICLE}

Rymar OD, Mustafina SV, Alferova VI, Denisova DV. Epidemiological studies of iodine deficiency in Novosibirsk: data of 25-years observation. Clinical and experimental thyroidology. 2020;16(2):4-11. doi: https://doi.org/10.14341/ket12539 Received: 20.07.2020. Accepted: 24.08.2020. Published online: 07.10.2020 